\title{
Mutation du travail et doctrine de prévention
}

Auteur.e.s : Alexis CHAMBEL, Valerie PUEYO, Pascal BEGUIN. Laboratoire EVS (UMR 5600)

alexis.chambel1@univ-lyon2.fr, valerie.pueyo@univ-lyon2.fr, pascal.beguin@univ-lyon2.fr

Thèse financée par le Parcours Doctoral National en Santé Travail coordonné par l'EHESP dans le cadre du Plan Santé Travail 3

\section{(1) Introduction}

Cette thèse débutée au 1er octobre 2019 marque la continuation d'une action de recherche, réalisée pour la DGT dans le cadre du PST3 (Plan Santé Travail 3), qui a porté sur la prévention des risques professionnels des aides à domicile salariés de « particuliers employeurs ») (Pueyo, Ruiz, et al., 2019). Ce travail préliminaire a
interroger ce qu'on peut désigner comme une « doctrine de prévention » (ibid.) actuellement mobilisée pour penser, cadrer et agir la prévention.

Un travail de conceptualisation est depuis opéré autour de la doctrine de prévention à partir de la notion de « dispositif » dans la philosophie de Foucault (1977). Celui-ci peut être défini susccinctement comme un ensemble d'éléments hétérogènes qui font système. La nature des éléments peut être extrêmement variable : « des discours, des institutions, des amenagements architecturaux, des decisions réglementaires, des lois, des mesures administratives, des enoncés scientifiques, des propositions philosophiques, morales, philanthropiques, bref : du dit, aussi bien que du non-dit " (ibid.), le dispositif étant le système formé par ces éléments. Parler de systèm en avant la place de certains éléments au coeur de la doctrine, autrement dit une partie de ces « jeuк».

Un dispositif émerge dans un contexte d'urgence, pour répondre à un objectif spécifique, et par extension oriente l'action. Celui que représente la doctrine de prévention a émerge au milieu du XiXème siecle en réponse à la dégradation de l'etat de sante des populations ouvrières. Il $s$ 'est ainsi historiquement construit et

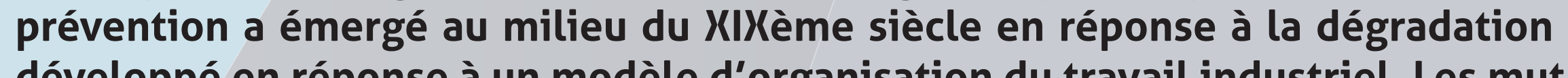
viennent requestionner la doctrine de prévention en place.
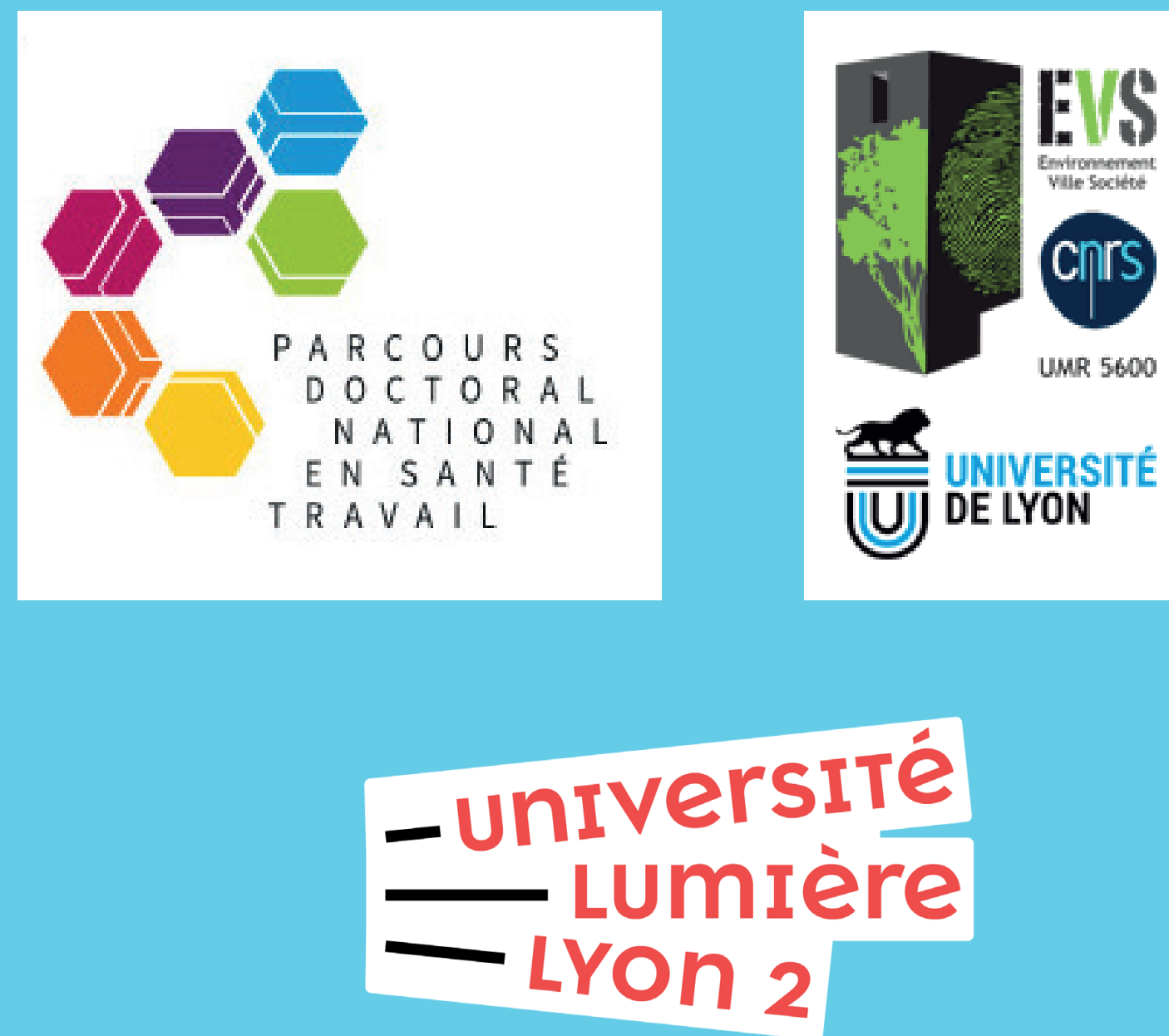

L'objectif de ce travail de recherche est d'identifier les points de dissonance entre la doctrine de prévention en place et les activités de service.

La doctrine de prévention pourrait être imaginée comme un prisme au travers duquel est observé le réel.

Elle en permet le découpage et une compréhension.

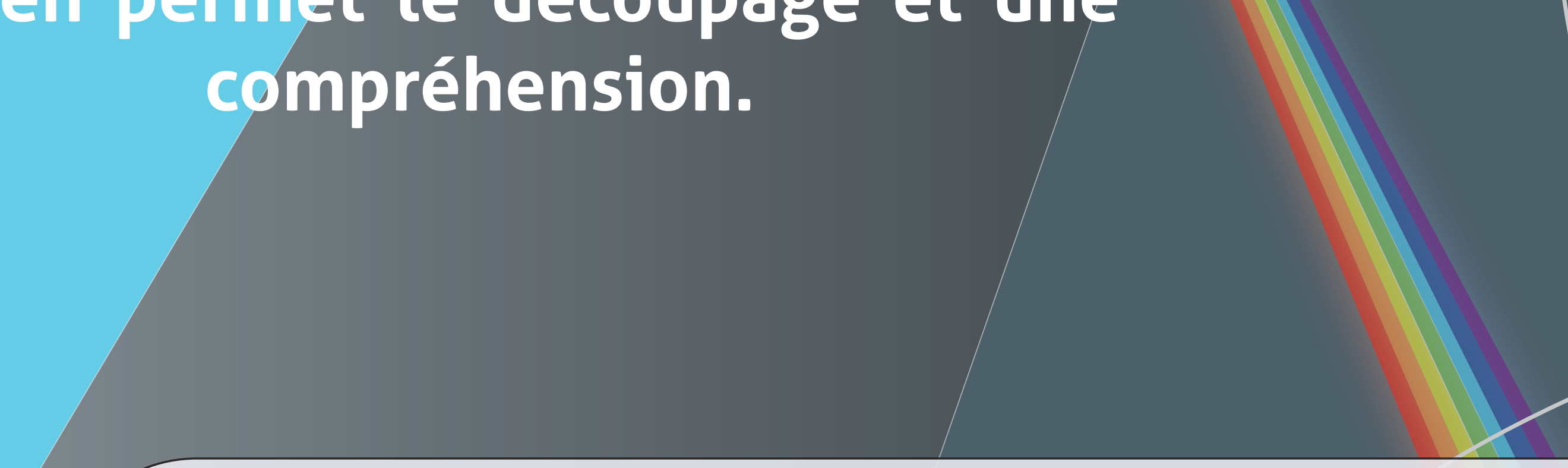

4 Les éléments structurants danger-risque-exposition relève de cette catégorie.

Nous nous attarderons plus spécifiquement sur le concept d'exposition qui opère le lien entre le dange

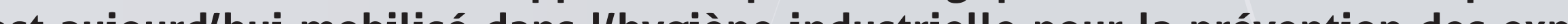

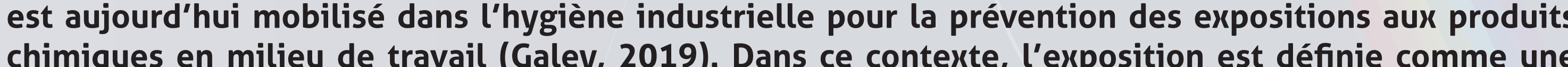
(a)

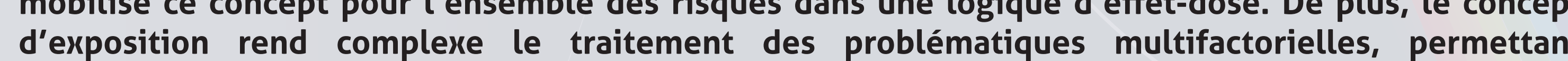
difficilement la prise en compte des interactions et de la situation (Dallat et all, 2017 cite par Galey (2019) p.50).
3 Les pôles de la santé et de la sécurité

Le pôle peut se définir comme étant un « point central qui exerce une attraction, un rayonnement » (CNRTL). Au cœu r de tout un sous-système d'éléments pouvant circuler plus ou moins aisément d'un pôle à l'autre de la doctrine et La sécurité se distingue par une « réalité de l'absence » (Cuvelier, 2011) : la doctrine de prévention porte une vision

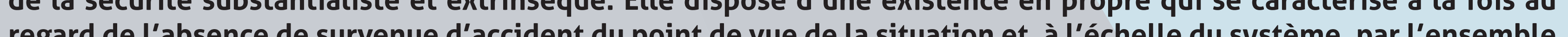
des éléments mis en place pour permettre de les éviter.

La santé présente quant à elle une certaine ambivalence. Si les textes et l'esprit s'inscrivent sans ambiguité dans un modele de la sante relevant de l'etat de complet bien-être dépendant du corps, de l'esprit et du social, il ne s'agit pas dans une dynamique qui, en toile de fond dessinel'ancrage de la pathologie La santé se défnit alors à l'image de la sécurité, par la négative dans les contours de l'absence de maladie ou d'infirmité (Pueyo, Ruiz, et al., 2019). L'articulation entre les deux champs représentent une zone floue de la doctrine de prévention, à la fois relativement

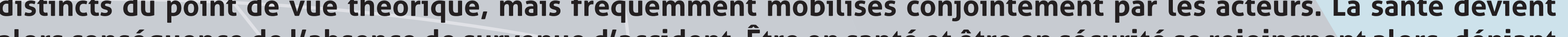

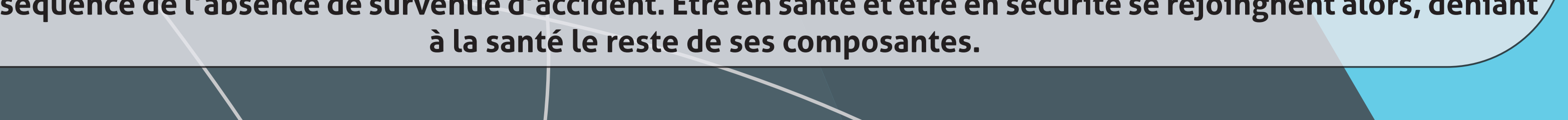

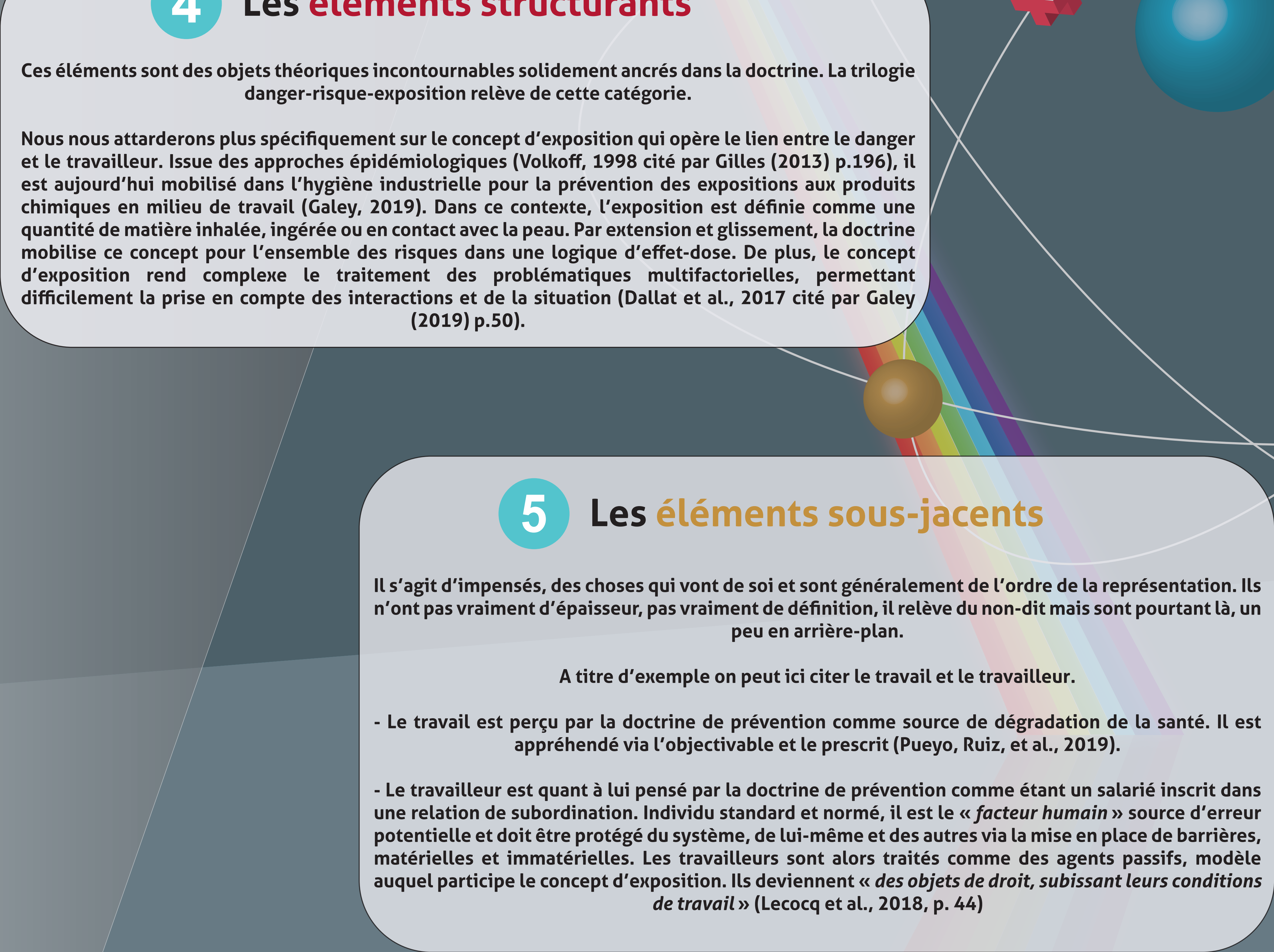

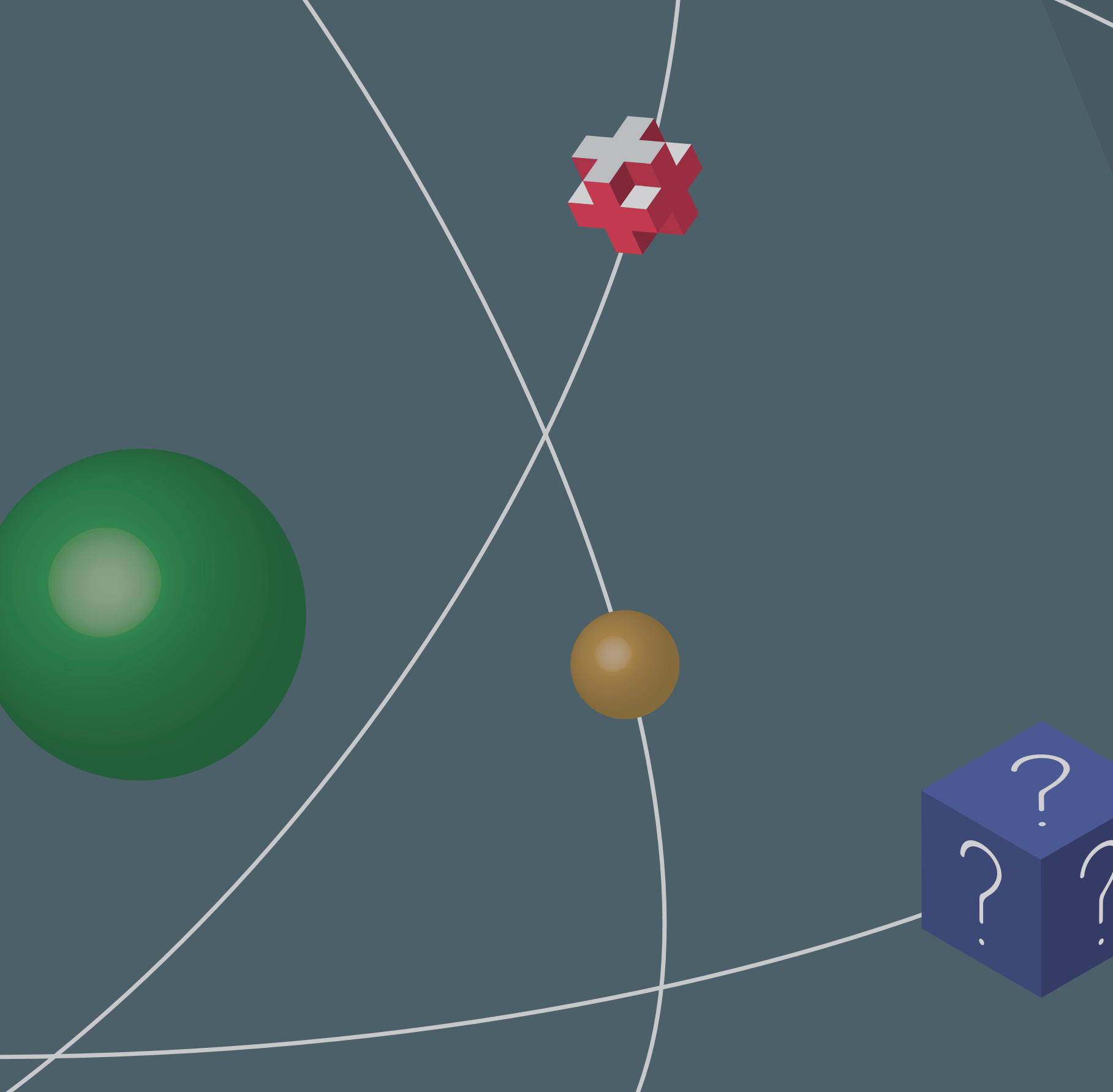

6 Une conceptualisation en cours Ce travail de recherche se poursuit, d'autres « jeux» (Foucault, 1977) se démarquent comme par exemple:

(bes registres de connaissances et courant de pensee relèvent plutôt des « bornes de savoirs (ibid.) qui naissent du dispositif et le conditionnent. Ils marquent l'ensemble des autres Les schémas d'action mettent en mouvement les autres composantes du dispositif dans la . Ils donnent alors lieu à des

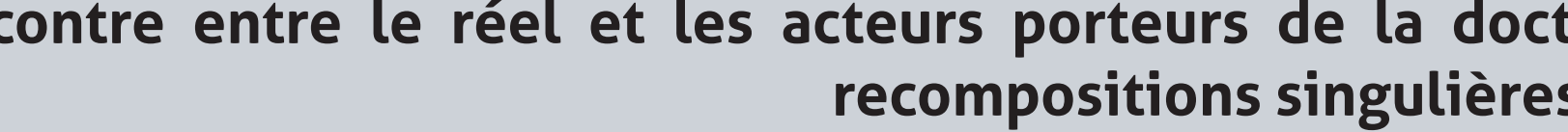

Les objets matériels et symboliques cristallisent les autres éléments dans le réel, leur donnan t une forme physique, comme dans un mécanisme d'agrégation.

Pour Vuillemin (2012) un dispositif se définit comme une « table d’opération », qui indique une orientation cohérente parce qu'il découpe un champ. Mais qui, inévitablement, laisse certains éléments dans l'ombre

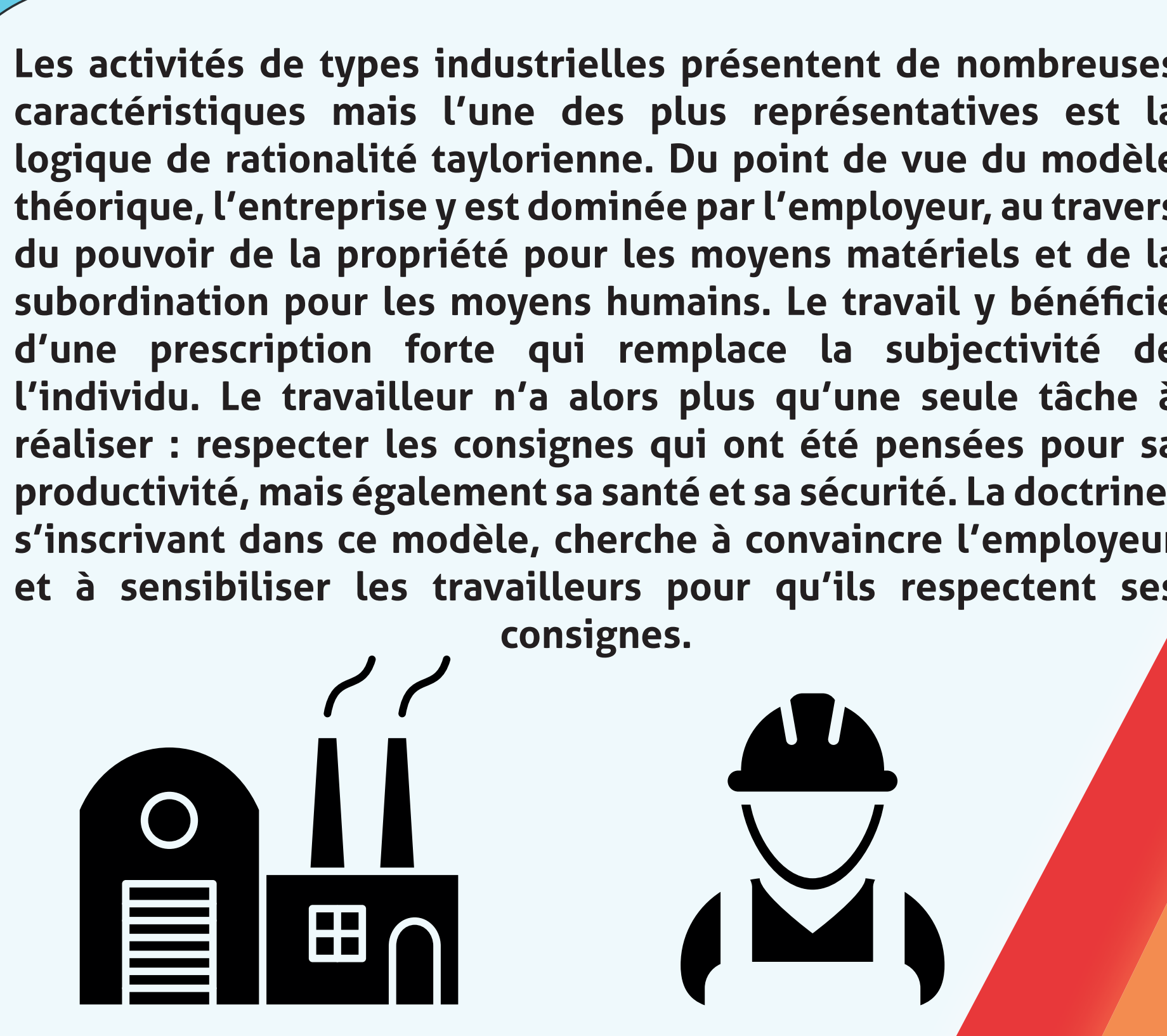

Activités industrielles

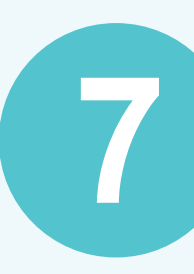

Activités servicielles

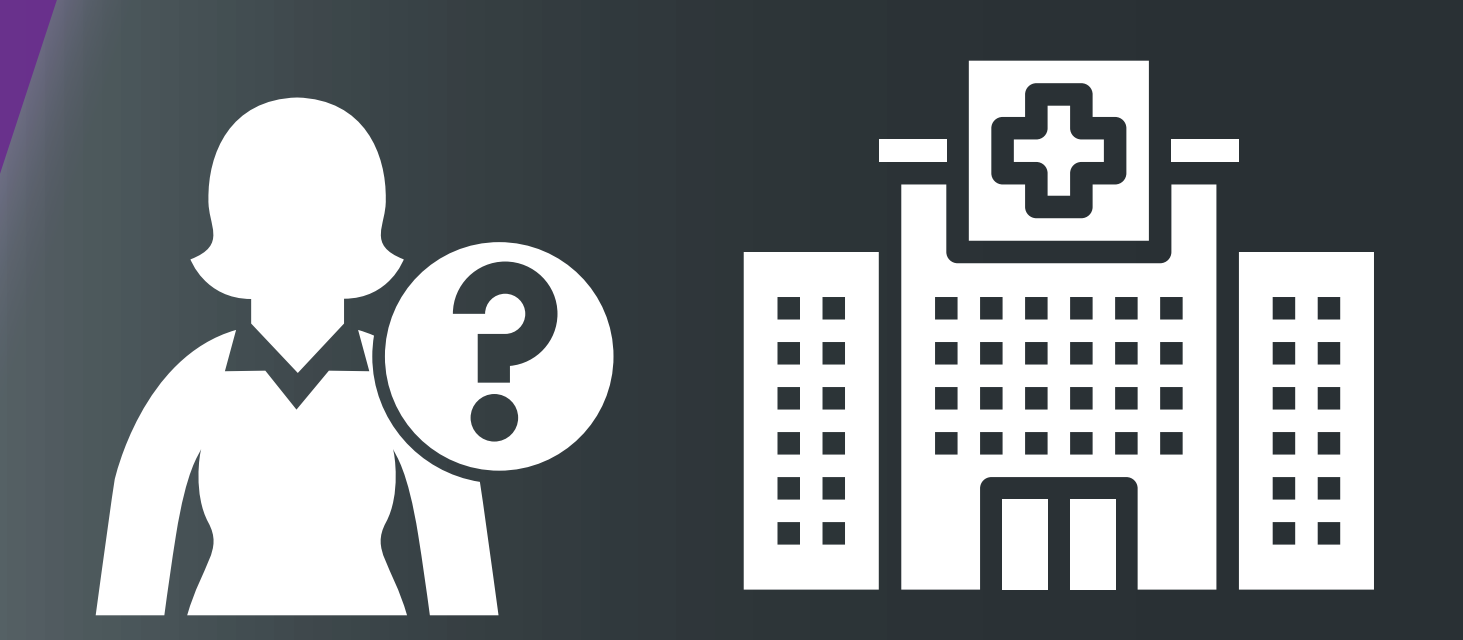

Les activités de types servicielles possèdent des caractéristiques qui débordent le périmètre appréhendé
par la doctrine de brente par la doctrine de prévention. L'eenemple le plus
représentatifi est le benéficaire. Coproducteur du service

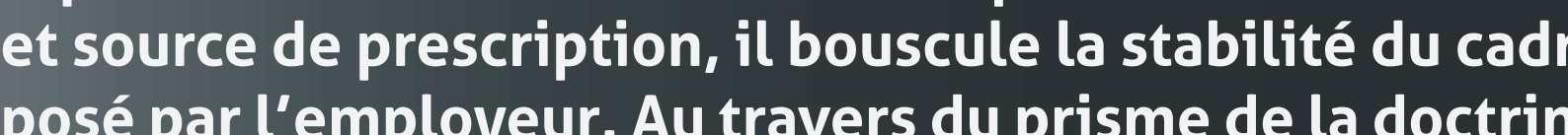

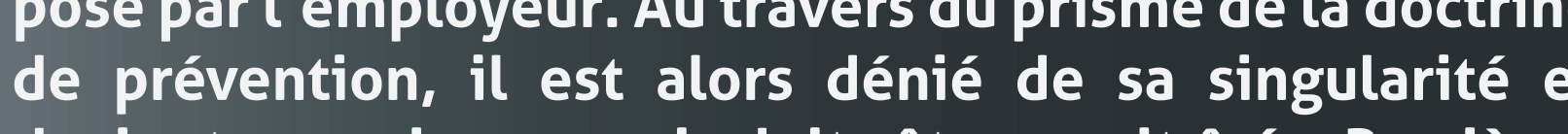
devient un risque qui doit être maitrise. Barrèeres
matérielles et organisationnelles seront déployées pour

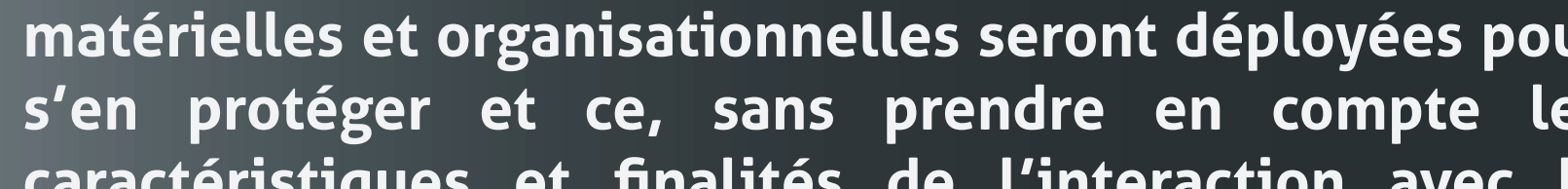

\section{Méthodologie}

Afin de réinterroger les points de dissonance entre la doctrine de prévention en place et les activités de service, plusieurs projets de prévention sont conduits dans des activités de services
différentes.

L'objectif n'est est d'être réprésentatif de l'ensemble des activités de service mais d'en choisir qui s'éloignent du modèle industriel selon différents paramètres.

Le point de vue de la doctrine de prévention est porté par des acteurs de la prévention qui son soit parties intégrantes du projet, soit interrogés à la suite de ces projets sur les actions qui on été déployés.

cours aupès d'une
médiation sociale.

\section{Bibliographie}

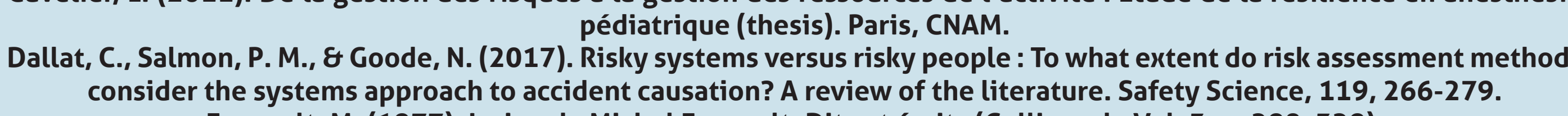

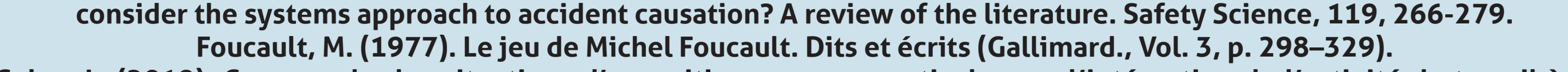

Gilles, M. (2013). Compter pour peserø: La

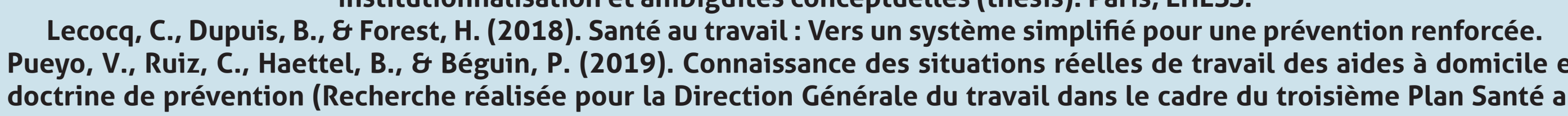

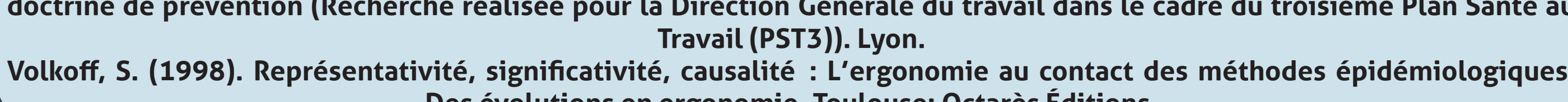

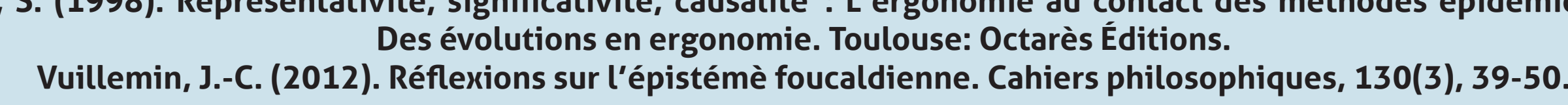

